PESHAWAR JOURNAL OF PSYCHOLOGY AND BEHAVIORAL SCIENCES, 2017, VOL. 3, NO. 1, 1-19

\title{
Role of Mindfulness and Psychological Wellbeing Between External Locus of Control and Depression: A Moderated Mediation Model
}

\author{
Sadia Niazi ${ }^{1}$ and Adnan Adil ${ }^{2}$ \\ University of Sargodha
}

The present research aimed at examining the role of mindfulness and psychological wellbeing in the relationship between external locus of control and depression. Five Facet Mindfulness Questionnaire (Baer, 2003), Ryff Scale of Wellbeing (Ryff, 1989), DAAS Depression Subscale (Lovibond \& Lovibond, 1995), and Rotter's Locus of Control Scale (Rotter, 1966) were administered on a convenient sample of three hundred and four $(\mathrm{N}=304)$ adults of Sargodha city (Punjab, Pakistan) to operationalize mindfulness, psychological wellbeing, depression, and external locus of control respectively. Results revealed that external locus of control had positive relationship with mindfulness whereas mindfulness and psychological wellbeing were negatively related with depression. Mindfulness fully mediated between external locus of control and depression. Psychological wellbeing moderated between mindfulness and depression by dampening their negative relationship. Moderated mediation analysis was undertaken through PROCESS macro for SPSS (Hayes, 2013), which revealed that conditional indirect relationship of external locus of control with depression was significant only at lower to moderate levels of psychological wellbeing. Implications of the study and suggestions for further research were discussed.

Keywords: mindfulness, external locus of control, psychological wellbeing and depression

Mindfulness is present-centered awareness and practice of focusing on the reality of the present moment without becoming engaged in elaborative thoughts or emotional reactions (Carlson, Speca, Patel, \&

\footnotetext{
${ }^{1}$ Lecturer, Department of Psychology, University of Sargodha

${ }^{2}$ Assistant Professor, Department of Psychology, University of Sargodha
} 
Goodey, 2003). It operates on individual's psychological wellbeing and there are numerous studies which reported changes in experience of emotions due to mindfulness such as mood particularly depression (Brown, Ryan, \& Creswell, 2007). Grob (2000) suggested that a person who focusses on external factors for failure and blame others is likely to be low on mindfulness with elevated levels of low mood. This is true because low mood or depression is often a corollary to feelings of powerlessness in a given situation, which are usually harbored by lack of mindfulness in people who are predominantly externally controlled. This line of reasoning has also been supported by Diener (2000) who proposes that individuals with external locus of control and low levels of mindfulness are depressive people and their depression level can be ameliorated through healthy psychological wellbeing. These streams of research warrants a study which can empirically test the role of mindfulness and psychological wellbeing in the relationship between external locus of control and depression and the present study is an endeavor towards the same end.

Anderson, Maes, and Berk (2010) claimed that the people who mainly cares about external factors and blame others for failure are less likely to focus on reality owing to which they are low on mindfulness, which may lead to depression. Within the framework of divergence theory, Stocks, April, and Lynton (2012) proposed that people having low levels of mindfulness have high external locus of control which is leading cause of depression. These studies suggest that external locus of control has unique relationship with mindfulness and depression, which can be influenced by psychological wellbeing. The present study is unique in the sense that it postulates and tests a theoretical model that links these variables into a single coherent theoretical framework. This model is depicted in Figure 1.

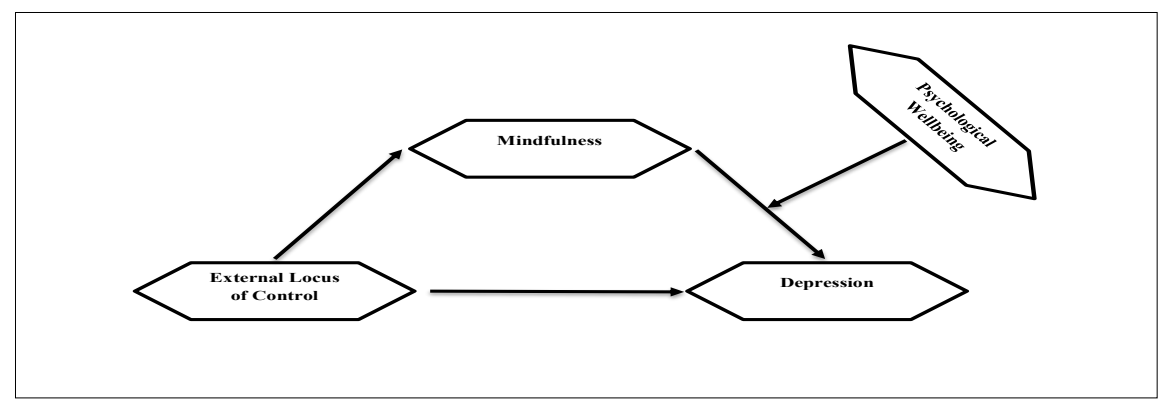

Figure 1. Conceptual framework of the present study. 
As evident in Figure 1, the present study assumes that external locus of control will have direct as well as indirect relationship with depression via mindfulness. Furthermore, the relationship of mindfulness with depression will be moderated by psychological wellbeing. The following section presents hypotheses of this investigation and briefly reviews the theoretical and empirical support for this conceptual framework.

Dyrbye, Thomas, and Shanafelt (2006) stated that people having good psychological health are less prone to depression. They also suggested that the people who can't actively take control of their lives and can't manage their problems are more likely to have mental issues such as depression. Beck, Brown, Berchick, Stewart, and Steer (1990) claimed hopelessness another leading cause of depression, which arises form one's beliefs that one cannot actively control or shape one's destiny. They stated that hopeless person attribute his failure to external factors because according to him, he/she has no control over them which leads them towards depressive thoughts. These findings are suggestive of the positive relation between external locus of control and depression and provide support for our first hypothesis.

Hypothesis 1: External locus of control will be positively related with depression. Depression or psychological distress and psychological wellbeing are not necessarily the opposite poles of a single continuum. Emphasizing the need for further research(Winefield,2012).

Gill, Taylor, and Pilkington (2012) concluded in their study that psychological wellbeing and psychological distress are not the opposite ends of the same underlying construct. This is in line with Watson, Clark, and Tellegen's (1988) conceptualization of positive affect (a primary component of psychological wellbeing) and negative affect (an important element of depression) as two independent dimensions of an individual's propensity of experiencing positive and negative moods. Therefore, the present study has operationalized both depression and psychological wellbeing as independent albeit negatively related constructs.

There is growing body of research literature suggesting mindfulness and psychological wellbeing as promoter of successful coping in stressful situations, and thus, they may serve as a buffer against depression. Brown et al. (2007) reported mindfulness and psychological wellbeing as positive variables, which may bring positivity in life. They 
suggested positive implications of mindfulness for psychological health by emphasizing the role of healthy emotional functioning in establishing and maintaining healthy life without depression. Depression may stem from prolonged stress that can negatively influence individual's cognitive and behavioural efforts used for managing internal and external demands of stressful situation. This stressful situation can be handled through emotional regulation by using mindfulness as coping strategy, which has been consistently related with psychological wellbeing (Brown-Iannuzzi, Adair, Payne, Richman, \& Fredrickson, 2014).

In another study, Ryan and Deci (2000) proposed that mindfulness is important in disengaging individuals from automatic thoughts, habits, and unhealthy behaviour patterns and thus could play a key role in promoting informed and self-endorsed behavioural regulation, which has long been associated with well-being enrichment. These studies are demonstrating the influential role of mindfulness and psychological wellbeing in relation to depression and provide impetus for the second hypothesis of the present study:

Hypothesis 2: Mindfulness and psychological wellbeing will be negatively related to depression. The prevailing view of the relationship between external locus of control and depression holds that individuals who define events in their lives as outside their control will be less able to cope effectively with stress, and therefore, more likely to experience physical and psychological distress (Brown et al., 2007). Stock (2012) proposed that externally controlled people are less mindful people. He argued that they are not open minded and self-regulated that's why they have more chances to face depression in their lives. This line of reasoning suggests that externally controlled individuals are unlikely to be mindful, which give rise to the third hypothesis of the present study:

Hypothesis 3: External locus of control and mindfulness will be negatively related. The indirect effect of external locus of control via mindfulness becomes meaningful given that external locus of control is assumed to be the predictor of mindfulness and depression, and mindfulness as predictor of depression. Thus, mindfulness may play a mediating role between external locus of control and depression. This proposition is supported by Fiori, Brown, Cortina, and Antonucci (2006) who suggested that people who let other to control their lives by losing their own control are externally oriented people and less mindful and this 
low level of mindfulness creates depressive thoughts. Therefore, we postulated:

Hypothesis 4: Mindfulness will mediate between external locus of control and depression. Kabat-Zinn (2009) has theorized psychological wellbeing as individual's ability for coping with stress by creating positive emotions. He claimed that psychological wellbeing helps in controlling negative emotions towards external factors especially of those people having external locus of control. Similarly, Beach (2001) proposed that psychological wellbeing equips an individual with an ability to communicate effectively and identify internal and external factors in more flexible and positive ways, which is conducive to the development of positive feelings. He further asserted that for externally oriented people, psychological wellbeing may serve as strong protective shield and may significantly mitigate their vulnerability to depression. Therefore, the next hypothesis of the present study proposes:

Hypothesis 5: Psychological wellbeing will moderate between mindfulness and depression by dampening their negative relationship. A concurrent reading of fourth and fifth hypotheses of the present study reveals the plausibility of moderated mediation because the moderated path in the sixth hypothesis also constitutes part of indirect effect of external locus of control on depression through mindfulness. This is suggestive of second order moderated mediation. This proposition is quite plausible and makes sense on theoretical and empirical grounds. Having an external locus of control involves a belief that one's destiny is not in one's own hands and external factors such as fate, the significant others, and luck may be more influential in determining the outcomes of one's efforts. This belief may spawn feelings of helplessness owing to which one is no more focusing on the reality of the present moment and depression may ensue. However, for individuals having the capability of coping with distress by creating positive emotions, this chain of events may not hold true. Among such individuals, levels of psychological wellbeing are high which may safeguard against depression as a corollary to the lack of mindfulness. Thus we propose:

Hypothesis 6: The indirect relationship between external locus of control and depression through mindfulness will be significant at lower levels of psychological wellbeing.

PESHAWAR JOURNAL OF PSYCHOLOGY AND BEHAVIORAL SCIENCES, 2017, VOL. 3, NO. 1, 1-19 


\section{Method}

\section{Participants}

In order to determine the appropriate sample size for the present research, power analysis was undertaken through $\mathrm{G}^{*}$ Power 3.0. The analyses revealed that for multiple regression analyses based on three predictors with $\alpha=.05$, power of .95 , a small effect size of Cohen's f $2=$ .07 could reliably be assessed with $\mathrm{N}=250$. Based upon findings of the power analyses where small effects can be detected from a sample size of 250 to 275 , a convenient sample of $N=304$ adults from Sargodha city was personally recruited in the present study. For this purpose, 400 questionnaire booklets were distributed among participants out of which 340 were returned to the researcher with a response rate of $85 \% .36$ questionnaires were discarded because of missing responses and response set. Participants were approached at their houses, shops, and offices. Men and women had equal representation in the sample. The age range of the sample was 25 to 45 years $(\mathrm{M}=37.33, \mathrm{SD}=11)$ with minimum educational level of higher secondary school certificate.

\section{Instruments}

The present study used Urdu translated versions of psychometrically sound self-report measures of the focal constructs. Demographics like age, gender, family type, and education were recorded through demographic sheet. The details of questionnaires that were used in this study are as follows:

\section{Five Facet Mindfulness Questionnaire (FFMQ)}

Five Facet Mindfulness Questionnaire (Baer, 2003) was translated into Urdu for measuring mindfulness in the present study. Back translation procedure was followed for ensuring the semantic and contextual equivalence between the original and translated versions of the questionnaire. Firstly, the questionnaire was translated into Urdu by three independent bilingual experts. After that, the three Urdu translated versions were compared and differences between Urdu translations of various items were reconciled for ensuring the theoretical uniformity of items through committee approach comprising of two $\mathrm{PhDs}$ and one MPhil in psychology. Secondly, the reconciled Urdu translated version of the questionnaire was back translated into English for assessing the 
conceptual equivalence of the reconciled backwards translation and original version. Finally, Urdu translated version was compared with original English version through committee approach. This instrument consisted of four stable patterns of self-awareness and attitude. It comprised of 39 items that were rated on 5 - point scale $(1 \neg=$ never or very rarely true to $5=$ always true). Baer (2003) reported this scale to be internally consistent $(\alpha=.91)$.

\section{Psychological Wellbeing Scale}

Urdu translated version (Jabeen, 2012) of Ryff's (1989) Psychological Wellbeing Scale comprising of 42 items was used for measuring psychological wellbeing. The items were rated on 6-point Likert scale $(1=$ strongly disagree to $6=$ strongly agree). Ryff (1989) reported it as highly reliable scale having $\alpha=.91$. Jabeen (2012) reported that Urdu translated version had an impressive test-retest reliability coefficient for two weeks $(r=.84)$

\section{Depression Anxiety and Stress Scale (DASS)}

The DASS is a 42-item questionnaire that was developed by Lovibond and Lovibond (1995) and its Urdu translated version (Farooqi $\&$ Habib, 2010) was used in the present study. It includes three selfreport scales designed to measure the negative emotional states of depression, anxiety and stress. In the present study, subscale of depression was used which consisted of 14 items. Each item is scored from 0 (do not apply to me at all over last week) to 3 (apply to me at all over last week). The internal consistency of depression subscale in the normative sample was fairly high $(\alpha=.91$; Lovibond \& Lovibond, 1995). Farooqi and Habib (2010) also reported an impressive Cronbach's alpha of .94 for Urdu translation of depression subscale.

\section{Locus of Control Scale.}

Short version of Rotter Internal versus External Locus of Control Scale (Rotter, 1966) comprised of 13 items and its Urdu translated version (Sardar, 1998) was used for assessing global control expectancies of the participant on the internality-externality continuum. Items indicating an external control orientation were scored as 1 point and items indicating an internal control orientation were scored as 0 . In statement 2, 3, 4, 8, 9, 10,11, 12 and 13 option " $b$ " represented external 
orientation and scored as 1 . On the other hand in statement 1, 5, 6 and 7 option "a" was considered as representing internal locus of control and scored as 0 . Possible scores on the scale range from 0 to 13 . The testretest reliability of a week of its Urdu version was also impressive ( $\mathrm{r}=$ .84; Sardar, 1998).

\section{Results}

The data of the present study were analyzed by using the Statistical Product and Service Solution (SPSS) version 20. The scales used to measure the focal constructs of the presents study were assessed in terms of their internal consistency and descriptive statistics. The proposed hypotheses were tested through hierarchical regression analyses. Results are presented in Table 1 to 4.

Table 1 depicts mean and standard deviations of all variables analyzed in the present study. Table 1 also depicts internal consistency index (alpha coefficients) for all scales used in the present study and suggested that all scales achieved satisfactory alpha level. Locus of control is forced-choice scale and its reliability cannot by determined through computing alpha coefficient. For this purpose, test retest reliability of a week was determined in pilot study on a sample of $\mathrm{N}=60$ adults, which was $r=.93$. Table 1 also displays that actual and potential ranges of various instrument were identical. This suggests that there was no evidence of restricted range of responses. Measures of skewness and kurtosis are also suggestive that data on each scale were normally distributed.

Table 1

Descriptive and Psychometric Properties of Scale of Present Study $(N=$ 304)

\begin{tabular}{|c|c|c|c|c|c|c|c|}
\hline \multirow{2}{*}{ Variable } & \multirow{2}{*}{$M$} & \multirow{2}{*}{$S D$} & \multirow{2}{*}{$\alpha$} & \multicolumn{2}{|c|}{ Range } & \multirow{2}{*}{$S k^{a}$} & \multirow{2}{*}{$K u^{b}$} \\
\hline & & & & Actual & Potential & & \\
\hline DSD & 6.87 & 7.32 & .91 & $0-3$ & $0-3$ & 1.47 & 2.25 \\
\hline RPWS & 170.50 & 19.68 & .74 & $1-7$ & $1-7$ & -0.06 & 0.47 \\
\hline FFMQ & 123.34 & 12.89 & .73 & $1-6$ & $1-6$ & 0.26 & -0.38 \\
\hline
\end{tabular}


Questionnaire. ${ }^{a}$ Standard error of skewness $=.14$ standard error of kurtosis $=.27$

Findings in table 1 suggested significant correlations among variables of the present study. All correlations were in the expected direction. External locus of control was negatively related to mindfulness and psychological wellbeing and had a positive relation with depression. Psychological wellbeing and mindfulness were positively related to each other, however both of them had a negative relationship with depression.

Table 2

Zero Order Correlations Among Variables of the Present Study (N=304)

\begin{tabular}{lllll}
\hline Variables & 1 & 2 & 3 & 4 \\
\hline Depression & & $-.51^{* *}$ & $-.43^{* *}$ & $.17^{* *}$ \\
Psychological Wellbeing & - & - & $.55^{* *}$ & $-.27^{* *}$ \\
Mindfulness & - & - & - & $-.23^{* *}$ \\
External Locus of Control & - & - & - & - \\
\hline$* * p<.01$ & & &
\end{tabular}

Table 2 delineates standardized direct and indirect relationships among variables of the present study. Results revealed that external locus of control, mindfulness, and psychological wellbeing had significant direct relationships with depression. External locus of control positively whereas mindfulness and psychological wellbeing were negatively related with depression. Thus, the first two hypotheses of the present study were supported. External locus of control also demonstrated a significant negative direct relationship with mindfulness, which supported the third hypothesis of the present study.

The indirect relationship of external locus of control with depression through mindfulness was also noteworthy because $95 \%$ confidence interval for this indirect relationship did not include zero. The mediating potential of mindfulness was further ascertained by the significant Sobel's $Z$ test (Sobel's $Z=3.63, \mathrm{p}<.05$ ). The relationship between external locus of control and depression was significant $(\beta=$ $.17, \mathrm{p}<.01)$ in the absence of mindfulness in the model, however, the same relationship became non-significant after mindfulness was entered in the model $(\beta=.16 \mathrm{p}>.05)$. This provided evidence that mindfulness fully mediated the relationship between external locus of control and depression. Thus, our fourth hypothesis was also supported. 
Table 3

Standardized Direct and Indirect Effects of Predictors of Depression $(N=304)$

\begin{tabular}{|c|c|c|c|c|c|c|c|c|}
\hline \multirow[t]{2}{*}{ Outcome } & \multirow[t]{2}{*}{ Predictors } & \multicolumn{3}{|c|}{ Direct Effect } & \multirow[t]{2}{*}{ Predictors } & \multicolumn{3}{|c|}{ Indirect Effect } \\
\hline & & $B$ & $L L$ & $U L$ & & $\beta$ & $L L$ & $U L$ \\
\hline $\begin{array}{l}\text { Mindfulnes } \\
\mathrm{s}\end{array}$ & ELOC & $-.23 * *$ & -.34 & -.12 & \multirow{5}{*}{ ELOC } & \multirow{5}{*}{$.09^{\mathrm{a}}$} & \multirow{5}{*}{.03} & \multirow{5}{*}{.15} \\
\hline \multirow[t]{4}{*}{ Depression } & Mindfulness & $\begin{array}{l}- \\
1.3 * * *\end{array}$ & -2.0 & -.71 & & & & \\
\hline & ELOC & .16 & -.38 & .72 & & & & \\
\hline & Wellbeing & $\begin{array}{l}- \\
2.1 * * *\end{array}$ & -2.7 & -1.4 & & & & \\
\hline & $\begin{array}{l}\text { Mindfulness } \\
\mathrm{x} \text { Wellbeing }\end{array}$ & $.68 * *$ & .23 & 1.1 & & & & \\
\hline \multicolumn{9}{|c|}{$\begin{array}{l}\text { Note. ELOC }=\text { external locus of control. Wellbeing }=\text { psychological } \\
\text { wellbeing } \\
\text { a Sobel's } Z=3.63, p<.001 \text {. }\end{array}$} \\
\hline
\end{tabular}

Table 3 shows significant direct relationship of mindfulness and psychological wellbeing with depression $(\beta=.68, \mathrm{p}<.001)$ was suggestive of moderating role of psychological wellbeing between mindfulness and depression. This moderation is plotted in Figure 2, which delineated that the negative relationship between mindfulness and depression was much stronger for individuals who were low on psychological wellbeing. In other words, when psychological wellbeing was high depression was low and vice versa irrespective of the fact whether participants were high or low on mindfulness. Thus, psychological wellbeing may moderate to dampen the negative relationship between mindfulness and depression, which augmented our fifth hypothesis. 


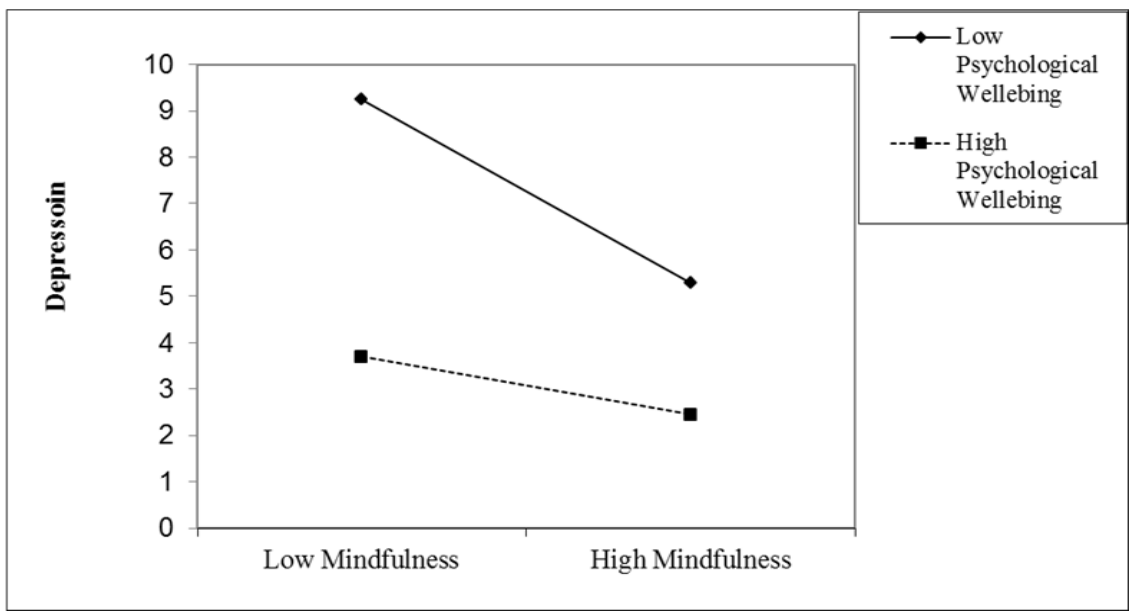

Figure 2. Moderating role of psychological wellbeing between mindfulness and depression.

This moderation might provide clues for the moderated mediation since the relationship between mindfulness and depression constituted part of the indirect path from external locus of control and depression. This moderated mediation was further investigated by computing the conditional indirect relationship of external locus of control with depression through mindfulness at various levels of psychological wellbeing through PROCESS macro for SPSS (Hayes, 2013). The results are presented in table 4.

Table 4

Conditional Indirect Effect of Mindfulness on Depression at Various Values of Psychological Wellbeing $(N=304)$

\begin{tabular}{lcccc}
\hline Mediator & $\begin{array}{c}\text { Moderator } \\
\text { (Wellbeing) }\end{array}$ & $B$ & $U L$ & $L L$ \\
\hline Mindfulness & -.13 & .52 & .19 & .94 \\
& -.6 & .42 & .15 & .74 \\
& .02 & .31 & .12 & .56 \\
& .73 & .19 & .07 & .40 \\
& 1.39 & .09 & -.03 & .27 \\
\hline
\end{tabular}

Note. Values for quantitative moderators are 10th, 25th, 50th, 75th, and $90 t h$ percentiles. 
The conditional indirect relationship of external locus of control with depression through mindfulness at various values of psychological wellbeing revealed that the indirect relationship was stronger when psychological wellbeing was low. The confidence intervals for the indirect relationship included zero when psychological wellbeing was high at 90th percentile; however, it did not contain zero at moderate and low values of psychological wellbeing (10th, 25th, 50th and 75th percentile). Thus, the meditational role of mindfulness between external locus of control and depression was more pronounced when psychological wellbeing was low. Thus, our sixth hypothesis was also supported.

\section{Discussion}

The present study aimed at finding relationship among psychological wellbeing, depression, mindfulness, and external locus of control. This study also proposed and tested model of depression specifying mindfulness as the mediator and psychological wellbeing as the moderator of the relationship of mindfulness and depression.

The results of the present study indicated significant correlations among mindfulness, psychological wellbeing, depression, and external locus of control. These findings are study is supporting the first hypothesis of the present study. These findings are supported on several fronts such as according to Wilber (2000), the philosophical, spiritual, and psychological traditions emphasize the importance of mindfulness in creating psychological wellbeing in life. He emphasized upon the significance of consciousness in human wellbeing, which could be

achieved through mindfulness. This viewpoint indicated that being conscious about the present life and accurate perception of reality is very important for achieving psychological wellbeing in life. Kabat-Zinn (2009) proposed that experiencing mindfulness plays an import role in facilitating psychological wellbeing. Rayan and Deci (2000) determined that mindfulness helps an individual to disengage from automatic thoughts, habits, and unhealthy behavior. These healthy behaviors may play key role in self-endorsed behavioral regulation, which has long been associated with wellbeing and personal enhancement.

In line with the first hypothesis of the present study, our results demonstrated that external locus of control was positively linked with depression. This finding is in line with literature relating external locus 
of control with dissatisfaction in life. Ganji and Navabinezhad (2012) proposed that there are abundant signs of criticism, blame, insult, humiliation, threat, and punishment in adults who are externally controlled. Externally controlled individuals are less likely to see their own flaws in everyday life conflicts. They tend to blame other people for each and every problem, which mostly leads to depression.

Results of the present study also supported our second and third hypotheses as it was observed that mindfulness and psychological wellbeing were negatively related to depression and externally controlled individuals were less likely to be mindful. Mindfulness is non-evaluative awareness about self and environment. It is thinking about facts and events without comparison and accurate observation of one's own psychological responses to external and internal stimuli (Carlson, Speca, Patel, \& Goodey, 2003). Carlson et al. (2003) asserted that a person having external locus of control will not think in that manner. His/her judgment about events will not be reality based. It will be one sidedjust blaming the external factors. This line of reasoning suggests that people having high external locus of control are less mindful people and they are more vulnerable to stress leading to depression.

The negative relationship between psychological wellbeing and depression is also grounded in Ryff's (2014) conceptualization of psychological wellbeing as global happiness linked with higher levels of purpose, self-acceptance, and positive relations in individual's life. He suggested that people high on psychological wellbeing view each and everything in positive manner because high psychological wellbeing is negotiation of life challenges in positive manner that may lead to improvements in psychological process resulting in reduced levels of stress and depression.

Results of the present study elucidated that mindfulness also fully mediated the relationship between external locus of control and depression which supported our fourth hypothesis. The mediating mechanism of mindfulness between external locus of control and depression can be cogently explained in terms of Bear's (2003) assertion, who referred to external locus of control as external acknowledgment of success and failure. This external acknowledgment leads toward blaming external factors and poor evaluation of internal and external aspects of life. This poor judgment is essentially reflected in lack of mindfulness. This line of reasoning is also espoused by Evas and Segerstron (2010) who proposed that people having high external locus of control are 
judgmental and reactive to external factors which shows they are less mindful. This lack of mindfulness may make people more focused on negative content of thought, which lead them to depression. Bear (2003) claimed that less mindful people allow their thoughts to come in their mind repeatedly and stuck in them without having control on them. This consistent merging in negative thoughts may result in elevated levels of depression.

Findings of the present study supported our fifth hypothesis because psychological wellbeing moderated between mindfulness and depression. This moderating role of psychological wellbeing is supported by pertinent literature that suggests mindfulness and psychological wellbeing as conductive approach towards positivity. Reb, Narayanan, and Chaturvedi (2014) defined mindfulness as awareness and nonjudgmental acceptance of one's moment-to-moment experience. They described it as potentially effective antidotes against common forms of psychological distress. Germer, Siegel, and Fulton (2005) claimed that mindfulness has theoretical and empirical relation with psychological wellbeing in regulating depression.

The final hypothesis of the present study was also supported as psychological wellbeing was the second order moderator of the mediated relationship between external locus of control and depression through mindfulness. These findings revealed that the indirect relationship of external locus of control with depression through mindfulness was stronger when psychological wellbeing was low. These findings are supported by Teasdale et al. (2000) who claimed that people having external locus of control are less mindful people because they focus on external factors for evaluation. This can lead them towards depression. They proposed that risk of occurrence and relapse of depression due to negative thoughts especially among individuals having external locus of control is high in less mindful people. Less mindful people make negative judgment's and evaluation based on negative thoughts. But this negativity leading to depression can be reduced by high psychological wellbeing. This is exactly what we have found in the present study where Psychological wellbeing turned out to be catalytic in enhancing the efficacy of mindfulness in reducing depressive symptoms among people with external locus of control. This moderated mediation is also in line with the results of Drake, Duncan, Sutherland, Abernethy and Henry (2008) who found that mindful person always focuses on his/her present, remains aware and conscious about his/her thoughts but less mindful 
cannot due to high external locus of control. Nevertheless, they also claimed that mindful person is satisfied or happier for particular time period relevant to his mindfulness state. They further asserted that a psychologically healthy person having mindful state will have more potential to fight with depressive thoughts, which are basically originated due to external locus of control.

\section{Conclusion and Implications}

Overall, the present study has revealed that external locus of control was positively whereas mindfulness and psychological wellbeing were negatively related to depression. Furthermore, this study suggested that external locus of control was negatively related to mindfulness and mindfulness had negative association with depression. Thus, external locus of control not only demonstrated a direct positive relationship with depression, it also exhibited an indirect positive relationship with depression via mindfulness. Results of the present study also suggested that psychological wellbeing weakened the negative association between mindfulness and depression. In other words, our findings may suggest that an individual with an external locus of control is likely to be less mindful and more depressive; however, this elevated level of depression can be regulated by harnessing one's psychological wellbeing.

Our findings not only provided a theoretical framework for understanding the dynamic interplay between locus of control and mindfulness in relation to depression and psychological wellbeing, it also highlighted the practical significance of mindfulness training in alleviating depression and fostering wellbeing. Mindfulness training provides a viable option for successful coping with depression because besides being an acknowledged protecting factor against developing depression (Raveepatarakul, Suttiwan, Iamsupasit \& Mikulas, 2014), mindfulness training has an established positive influence on psychological wellbeing (Goyal et al., 2014) as well.

\section{Limitations and Suggestions}

The present study involves certain limitations, which should be considered while interpreting our findings. Use of cross-sectional design in the present study has ruled out the possibility of causal inferences about the influence of our focal constructs on depression. Nevertheless, the choice of these variables and their proposed relationships are justifiable in the light of pertinent theory and empirical research. Future 
studies should incorporate longitudinal design for determining the causal roles of mindfulness and external locus of control in relation to depression. Another methodological issue of the present study involved the use of self-report measures, which might have introduced common method variance resulting in inflated relationships. However, it should be noted that the variables of the present study can viably be operationalized through self-report measure. This is especially true about mindfulness, which constitutes the core construct of the present study. Finally, the sample of the present study was drawn from non-clinical adult population which is not representative of the clinically depressed population. Therefore, any generalization of our findings should be made with caution. Future studies should explore the role of locus of control and mindfulness in relation to various psychological disorders in clinical populations.

Further research should also continue to explore other potential applications of mindfulness and psychological wellbeing, and practical issues concerning the delivery, implementation, and dissemination of mindfulness oriented interventions should be examined. This understating and application of interventions can be helpful in alleviating human psychological sufferings and help us live a life that is happier and more fulfilling.

\section{References}

Anderson, G., Maes, M., \& Berk, M. (2012). Biological underpinnings of the commonalities in depression, somatization, and chronic fatigue syndrome. Medical Hypotheses, 78(6), 752-756.

Baer, R. A. (2003). Mindfulness training as a clinical intervention: A conceptual and empirical review. Clinical Psychology: Science and Practice, 10, 125-143.

Beach, S. R. (2001). Marital and family processes in depression: A scientific foundation for clinical practice. Washington DC: American Psychological Association.

Beck, A. T., Brown, G., Berchick, R. J., Stewart, B. L., \& Steer, R. A. (1990). Relationship between hopelessness and ultimate suicide: A replication with psychiatric outpatients. American Journal of Psychiatry, 147 (2), 190-195.

Brown, K. W., Ryan, R. M., \& Creswell, J. D. (2007). Mindfulness: Theoretical foundations and evidence for its salutary effects. Psychological Inquiry, 18(4), 211-237.

PESHAWAR JOURNAL OF PSYCHOLOGY AND BEHAVIORAL SCIENCES, 2017, VOL. 3, NO. 1, 1-19 
Brown-Iannuzzi, J. L., Adair, K. C., Payne, B. K., Richman, L. S., \& Fredrickson, B. L. (2014). Discrimination hurts, but mindfulness may help: Trait mindfulness moderates the relationship between perceived discrimination and depressive symptoms. Personality and Individual Differences, 56, 201-205.

Carlson, L. E., Goodey, Patel, K. D., \& E Speca, M., (2003). Mindfulness-based stress reduction in relation to quality of life, mood, symptoms of stress, and immune parameters in breast and prostate cancer outpatients. Psychosomatic Medicine, 65(4), 571581.

Diener, E. (2000). Subjective well-being: The science of happiness and a proposal for a national index. American Psychologist, 55(1), 34.43.

Drake, L., Duncan, E., Sutherland, F., Abernethy, C., \& Henry, C. (2008). Time perspective and correlates of wellbeing. Time \& Society, 17(1), 47-61.

Dyrbye, L. N., Thomas, M. R., \& Shanafelt, T. D. (2006). Systematic review of depression, anxiety, and other indicators of psychological distress among US and Canadian medical students. Academic Medicine, 81(4), 354-373.

Farooqi, Y., \& Habib, M. (2010). Gender difference in anxiety, depression and stress among survivors of suicide bombing. Pakistan Journal of Social \& Clinical Psychology, 8(2), 145-155.

Fiori, K., Brown, E. E., Cortina, K. S., \& Antonucci, T. C. (2006). Locus of control as a mediator of the relationship between religiosity and life satisfaction: Age, race, and gender differences. Mental Health, Religion and Culture, 9 (3), 239-263.

Ganji, A. A., \& Navabinezhad, D. (2012). The relationship between locus of control and marital satisfaction of couples. Life Science Journal, 9(4), 294-298.

Germer, C. K., Siegel, R. D., \& Fulton, P. R. (Eds.). (2013). Mindfulness and psychotherapy. Guilford Press.

Goyal, M., Singh, S., Sibinga, E. M., Gould, N. F., Rowland-Seymour, A., Sharma, R., \& Haythornthwaite, J. A. (2014). Meditation programs for psychological stress and well-being: a systematic review and meta-analysis. JAMA Internal Medicine, 174(3), 357368. 
Grob, A. (2000). Perceived control and subjective well-being across nations and across the life span. Culture and Subjective Wellbeing, 79 (3), 319-339.

Hayes, A. F. (2013). Introduction to mediation, moderation, and conditional process analysis: A regression-based approach. NY: Guilford Press.

Jabeen, A. S. (2012). Resilience and psychological distress in dengue patients. Unpublished Master thesis. Institute of Applied Psychology, University of the Punjab, Lahore.

Kabat-Zinn, J. (2009). Full catastrophe living: Using the wisdom of your body and mind to face stress, pain, and illness. NY: Bantam Books.

Lovibond, P. F., \& Lovibond, S. H. (1995). The structure of negative emotional states: Comparison of the Depression Anxiety Stress Scales (DASS) with the Beck Depression and Anxiety Inventories. Behavior Research and Therapy, 33 (3), 335-343.

Raveepatarakul, J., Suttiwan, P., Iamsupasit, S., \&Mikulas, W. L. (2014). A mindfulness enhancement program for 8 to 11 year-old Thai children: Effects on mindfulness and depression. Journal of Health Research, 28(5), 335-341.

Reb, J., Narayanan, J., \& Chaturvedi, S. (2014). Leading mindfully: Two studies on the influence of supervisor trait mindfulness on employee well-being and performance. Mindfulness, 5(1), 36-45.

Rotter, J. B. (1966). Generalized expectancies for internal versus external control of reinforcement. Psychological Monographs, 80 (1), 609.

Ryan, R. M., \& Deci, E. L. (2000). Self-determination theory and the facilitation of intrinsic motivation, social development, and wellbeing. American Psychologist, 55 (1), 68.

Ryff, C. D. (1989). Happiness is everything, or is it? Explorations on the meaning of psychological well-being. Journal of Personality and Social Psychology, 57 (6), 1069.

Ryff, C. D. (2014). Psychological wellbeing revisited: Advances in science and practice. Psychotherapy and Psychosomatics, 83 (1), 10-28.

Sardar, S. (1998). Study of relationships among childhood paternal loss, sex-role orientation, self-esteem and locus of control in male and female students. Unpublished Doctoral Dissertation. University of Karachi, Karachi, Pakistan. 
Stocks, A., April, K. A., \& Lynton, N. (2012). Locus of control and subjective well-being: A cross-cultural study. Problems and Perspectives in Management, 10 (1), 17-25.

Teasdale, J. D., Segal, Z. V., Williams, J. M., Ridgeway, V. A., Soulsby, J. M., \& Lau, M. A. (2000). Prevention of relapse/recurrence in major depression by mindfulness-based cognitive therapy. Journal of Consulting and Clinical Psychology, 68(4), 615-623.

Watson, D., Clark, L. A., \& Tellegen, A. (1988). Development and Validation of brief measures of positive and negative affect: The PANAS Scales. Journal of Personality and Social Psychology, 54 (6), 1063-1070.

Wilber, K. (2000). Integral psychology. Boston: Shambhala Publications. Winefield, H. R., Gill, T. K., Taylor, A. W. \& and Pilkington, R. M. (2012). Psychological well-being and psychological distress: Is it necessary to measure both? Psychology of Well-Being: Theory, Research and Practice, 2 (3), 1-14. 